\title{
Alterações fundoscópicas e sua associação clínica em pacientes com tuberculose, no Distrito Federal
}

Posterior eye lesions and their clinical association in patients with tuberculosis, in the Federal District, Brazil

Gustavo Federici Mendes ${ }^{1}$

Rachel Cortinhas Toríbio

Tomaz Aiza Alvares ${ }^{2}$

Rosicler Rocha Aiza Alvares ${ }^{3}$
RE S U M O

Objetivo: Avaliar a incidência de lesões de fundo de olho em pacientes com tuberculose no Distrito Federal e identificar fatores de risco para o aparecimento dessas lesões. Métodos: Em estudo prospectivo, foi realizada oftalmoscopia binocular indireta em 292 pacientes com tuberculose sistêmica. Estes pacientes foram avaliados em cinco diferentes Hospitais Regionais do Distrito Federal, entre 1ํde agosto de 1997 e 30 de julho de 1998. Análise multivariada foi aplicada para identificar associações entre a variável "lesão de fundo de olho" e as variáveis "sexo, idade, baciloscopia, HIV, localização da tuberculose, internação dos pacientes, tempo de sintomas, procedência e experiência anterior com tratamento". Resultados: Os resultados deste estudo mostraram incidência de 5,5\% de lesões fundoscópicas em pacientes com tuberculose. Análise multivariada mostrou que a co-infecção pelo HIV, internação dos pacientes e tuberculose miliar foram fatores de risco para a presença de lesões de fundo de olho. Quando essas características estiveram presentes simultaneamente, a probabilidade de um indivíduo apresentar estas lesões foi de $66 \%$. Conclusões: Na avaliação da incidência de lesões de fundo de olho em pacientes com tuberculose, devem ser levadas em consideração certas características individuais, visto que fatores de risco como a co-infecção pelo HIV, tuberculose miliar, e internação, influenciaram na presença destas lesões.

Descritores:Tuberculose/complicações;Fundodeolho; Coróide;Tuberculose ocular; Tuberculosemiliar;SíndromedelmunodeficiênciaAdquirida

I N T R O D U Ç ̃̃ O

A tuberculose é uma doença infecto-contagiosa causada pelo Mycobacterium tuberculosis ${ }^{(1)}$. Doença sistêmica, com predileção pelo pulmão, pode acometer vários órgãos do corpo humano por disseminação hematogênica ${ }^{(1)}$, e os bacilos, uma vez na corrente sangüínea, podem, ocasionalmente, atingir os olhos ${ }^{(2-3)}$.

A primeira lesão ocular presumível por tuberculose foi descrita por Maitre* em 1711 ${ }^{(4)}$. Koch $^{* *}$ em 1882 descobriu o bacilo ${ }^{(1)}$ e Haab ${ }^{* * *}$ em 1884 demonstrou este microorganismo nos tecidos oculares ${ }^{(4)}$.

\footnotetext{
* Maitre JAN em 1711 apud Duke Elder S, Abrams D. Diseases of uveal tract. In: Duke Elder. System of Oftalmology. St. Louis: CV Mosby; 1970. p.246-84.

** Robert Koch em 1882 apud Brasil. Ministério da Saúde: Campanha Nacional Contra Tuberculose. Controle da tuberculose: uma proposta de integração ensino serviço. Brasília; 1994.

*** Haab em 1884 apud Duke Elder S, Abrams D. Diseases of uveal tract. In: Duke Elder. System of Oftalmology. St. Louis: CV Mosby; 1970. p.246-84.
} 
No início do século XX, a tuberculose ainda era de alta letalidade, com uma sobrevida de apenas $40 \%$ após o diagnóstico. Com a descoberta dos agentes quimioterápicos na década de 40, os índices de mortalidade pela doença caíram drasticamente, e no final da década de 70 , esta foi considerada um problema praticamente solucionado ${ }^{(5)}$.

Nos últimos anos, a tuberculose vem ressurgindo, sendo a co-infecção com o Vírus da Imunodeficiência Humana (HIV) o mais potente fator de risco para o desenvolvimento da doença, favorecendo as formas extra-pulmonares e disseminadas ${ }^{(6)}$. Apesar da existência de poucos trabalhos na literatura sobre o assunto, pode-se esperar um aumento do número de casos de tuberculose ocular ${ }^{(2,7-10)}$.

Devido à falta de trabalhos, no Distrito Federal, sobre alterações oculares em pacientes com tuberculose, realizou-se um estudo prospectivo para avaliar a incidência de lesões de fundo de olho (FO) em atividade nestes pacientes, e identificar fatores de risco para o seu aparecimento.

\section{MÉ TODOS}

Realizou-se um estudo prospectivo, no qual foram examinados 292 pacientes com tuberculose sistêmica, independente da presença de sintomas oculares, no período de 1ํ de agosto de 1997 a 30 de julho de 1998.

A avaliação ocular dos pacientes foi realizada em cinco diferentes Hospitais regionais do Distrito Federal, sendo examinados tanto pacientes em acompanhamento ambulatorial quanto internados em enfermaria.

A coleta dos dados foi feita em 2 etapas:

1) Coleta de dados relativos ao exame oftalmológico dos pacientes por avaliação do FO, com oftalmoscópio binocular indireto, após preenchimento de termo de consentimento livre e esclarecido. Os pacientes que apresentaram alterações fundoscópicas suspeitas de tuberculose foram encaminhados para exame da acuidade visual, biomicroscopia, medida da pressão intra-ocular (PIO) e angiofluoresceinografia. Pacientes que apresentaram alterações em um olho ou em ambos os olhos, foram considerados como sendo somente um caso. Só foram consideradas as lesões com processo inflamatório em atividade. As lesões cicatrizadas (tipo coriorretinites) foram excluídas para diminuir a possibilidade de erro diagnóstico.

2) Coleta dos dados relativos à doença (tuberculose sistêmica) obtidos da ficha de notificação compulsória da Coordenação de Controle da Tuberculose do DF e dos prontuários dos pacientes. Estas fontes continham informações sobre a doença descritas pelos médicos tisiologistas assistentes dos Hospitais Regionais e permitiram o preenchimento de um protocolo contendo as seguintes variáveis: a)localização da tuberculose: pulmonar, forma da doença que restringe-se ao pulmão; extrapulmonar, tuberculose localizada em outro órgão que não o pulmão; miliar, forma grave da doença com quadro infeccioso importante; $b$ ) baciloscopia, exame de escarro positivo ou negativo; c) HIV, sorologia positiva ou negativa; d) sexo; e) idade do paciente; f) internação, caso o paciente estivesse internado ou em acompanhamento ambulatorial; g) tempo de sintomas, antes do início do tratamento específico para tuberculose; h) procedência dos pacientes, se eram do DF ou de outro estado e i) experiência anterior com tratamento, se o paciente era caso novo ou retratamento.

$\mathrm{Na}$ análise dos resultados, foi realizada uma regressão logística multivariada para avaliar a associação entre a variável resposta "lesão de fundo de olho" com as variáveis independentes acima descritas. Para esse procedimento utilizou-se o programa estatístico SAS (Statistical Analysis System) versão $6.12^{*}$. O nível de significância considerado no estudo foi de $10 \%$, uma vez que, algumas variáveis independentes além da relevância estatística são consideradas importantes do ponto de vista médico** .

RESULTADOS E DISCUSSÃO

Foram encontradas lesões de FO em 20 olhos de 16 pacientes $(5,5 \%)$. Em 9 pacientes, as lesões ocorreram somente no olho direito $(56,3 \%)$, em 3 pacientes, no olho esquerdo $(18,7 \%)$ e em 4 , as lesões foram vistas nos dois olhos (25\%).

Em 14 pacientes $(87,5 \%)$ foram vistas lesões semelhantes a tubérculos de coróide (figuras 1 e 2). Em 11, essas lesões foram unilaterais $(78,5 \%)$ e em 3 , foram bilaterais $(21,5 \%)$. O número de nódulos na coróide variou entre 1 e 5 em 1 olho, chegando a 9 lesões em ambos os olhos de um único paciente. O tamanho das lesões variou entre 1/4 e 1 diâmetro de papila, foram mais freqüentes no pólo posterior, apresentaram coloração amarelada e bordas irregulares e, no exame de angiofluoresceinografia, mostraram hipofluorescência precoce (figura 3) com hiperfluorescência tardia (figuras 4 e 5). Dois pacientes $(12,5 \%)$ com tubérculos de coróide apresentaram estas mesmas lesões na cabeça do nervo óptico, acompanhadas de papilite (tabela 1). Os tubérculos de coróide são bem descritos na literatura,

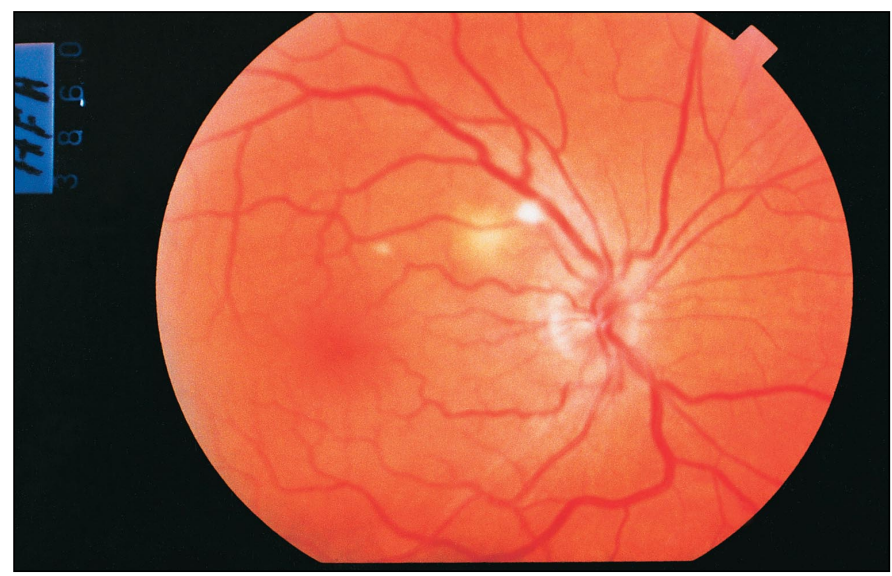

Figura1-Lesãodeconóideematividadeemumpacientecomtuberculose

* SAS. Institute Inc. Release 6.12. Cary, North Carolina, 1995.

** Hosmer DW, Lemeshow S. Applied logistic regression. New York: Wiley; 1998 


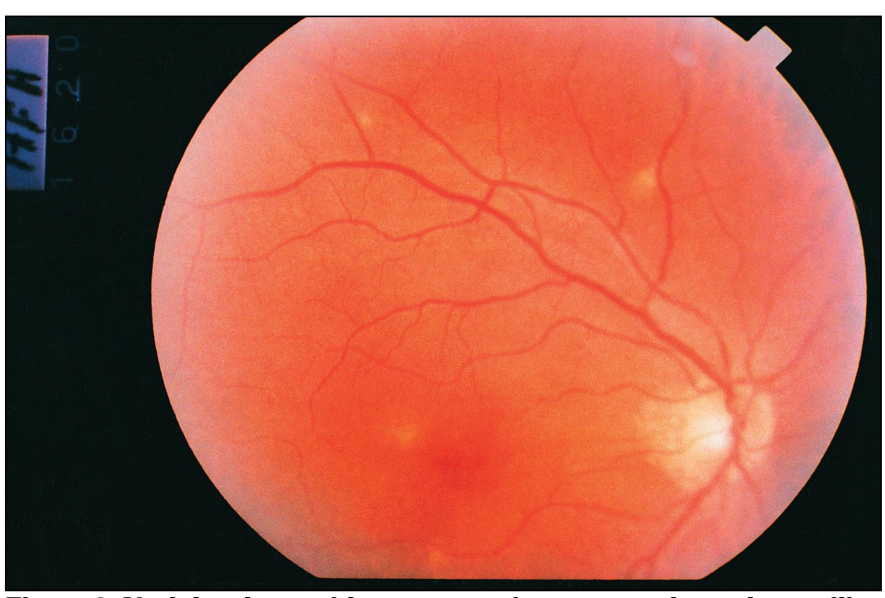

Figura 2-Nódulos decoróideemum pacientecomtuberculosemiliar

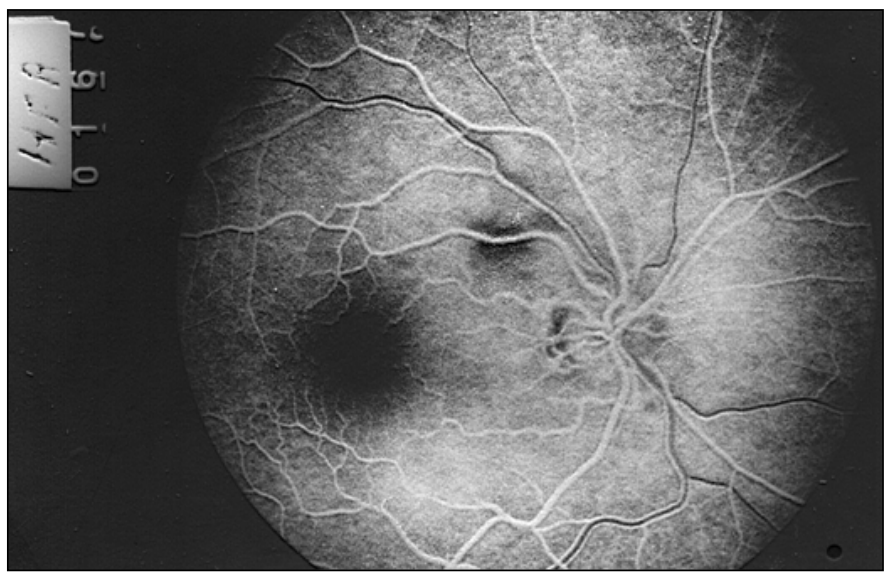

Figura 3-Angiofiluoresceinografia do paciente dafigura 1, mostrando hipofiuorescência da lesão nas fases iniciais doexame

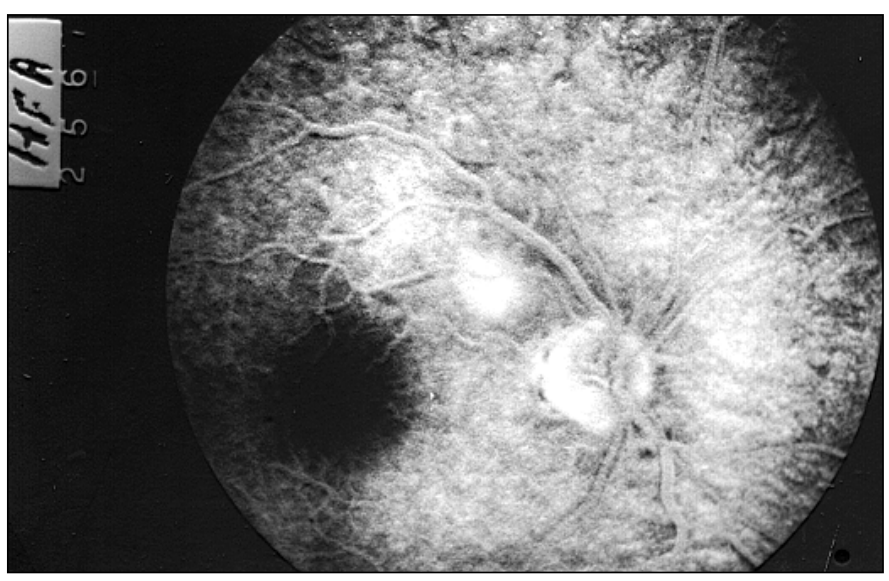

Figura 4-Angiofluoresceinografia dopaciente dafigura 1, mostrando hiperfiluorescêncianas fasestardiasdoexame,confirmandoapresença deumprocessoinflamatórioematividade

apresentam características clínicas bem definidas e segundo alguns autores, são um bom indicativo da disseminação hematogênica da tuberculose $\mathrm{e}^{(7-8,10-13)}$.

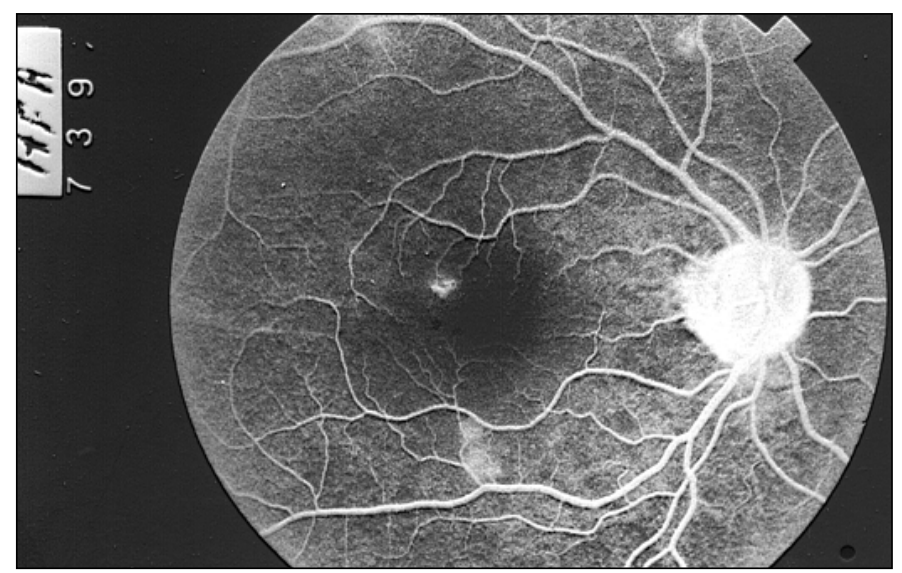

Figura 5-Angiofluoresceinografia do paciente dafigura 2, mostrando hiperfiuorescêncianasfasestardiasdoexame, confirmandoapresença deumprocessoinflamatórioematividade

Um paciente apresentou um tuberculoma em olho esquerdo, de aproximadamente 2,5 diâmetros de papila, acompanhado de intensa celularidade vítrea (tabela 1). Um outro apresentou periflebite dos vasos da região macular e peripapilar de olho direito, acompanhada de papilite e celularidade vítrea moderada (tabela 1). Apesar de ocorrerem com menos freqüência, essas lesões são constantemente descritas na literatura relacionadas à tuberculose e geralmente vêm acompanhadas de processo inflamatório em outras estruturas do olho, podendo ser destrutivas e levar à baixa visual irreversível ${ }^{(14-15)}$. Jabbour et al. (1985) diagnosticaram um caso de tuberculose pulmonar, pela avaliação de um tuberculoma de coróide ${ }^{(14)}$; Lyon et al (1985) descreveram um caso de granuloma de coróide em um paciente com tuberculose pulmonar avançada ${ }^{(15)}$; Rosen et al. (1990) relataram 2 casos de vasculite retiniana relacionados com tuberculose sistêmica ${ }^{(16)}$.

Alguns meses após o início da terapia específica para tuberculose observou-se evolução das lesões de coróide para cicatrização (figura 6) ou até mesmo desaparecimento (figura 7), como descrito na literatura ${ }^{(11-12)}$.

Somente três pacientes com lesões de FO (18,75\%) apresentaram acuidade visual inferior a 20/25 quando corrigidos, discordando de Bouza et al (1997), que apontaram a baixa da acuidade visual (inferior a 20/25) como fator de risco para a tuberculose ocular ${ }^{(2)}$; e concordando com outros autores que encontraram lesões de coróide em pacientes com tuberculose, sem queixas de baixa visual ${ }^{(8,10,12-13,17)}$.

\begin{tabular}{|lcc|}
\hline \multicolumn{3}{|l|}{ Tabela 1. Lesões defundode olhocompativeis comtuberculose } \\
Lesão de fundo de olho & Freqüência & $\%$ \\
Tubérculos decoróide & 12 & 75,0 \\
Tubérculos decoróideepapilite & 2 & 12,5 \\
Tuberculomaevitreíte & 1 & 6,25 \\
Vasculiteepapilite & 1 & 6,25 \\
TOTAL & 16 & 100,0 \\
\hline
\end{tabular}




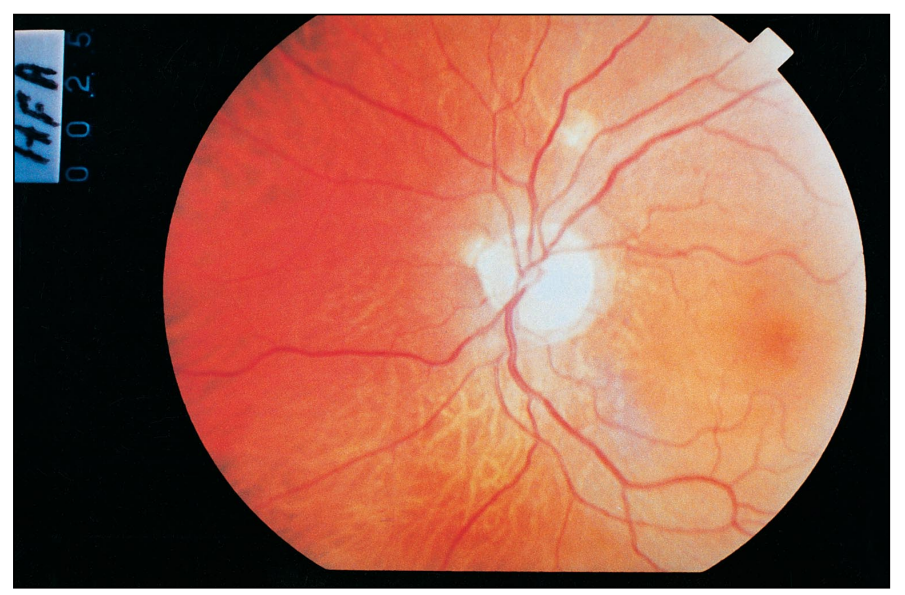

Figura 6-Nódulos de coróide alguns meses após o início da terapia específica paratuberculose, apresentandosinaisdecicatrização.Notar quea lesãotoma-seesbranquiçada comos bordos mais nítidos

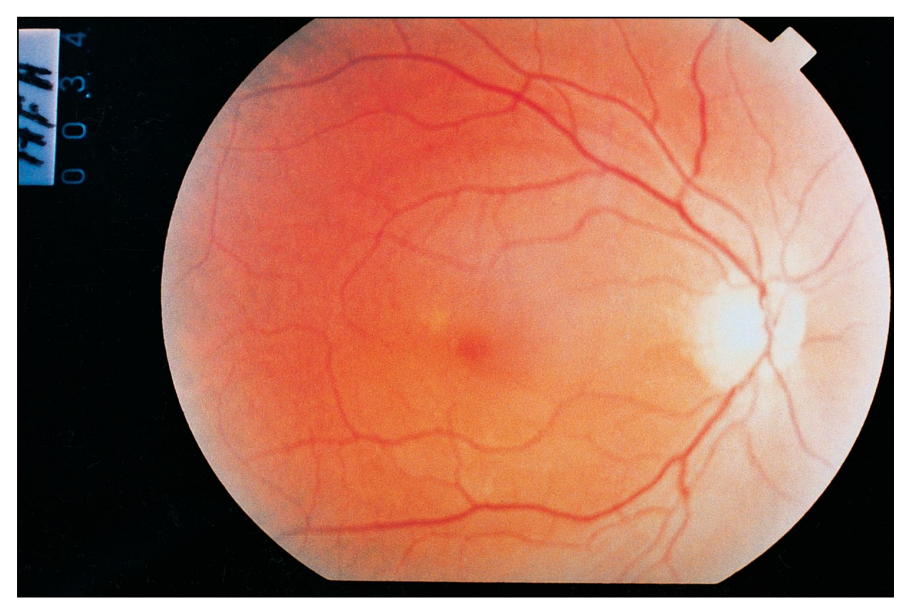

Figura 7-Retinocrafiadopacientedafigura2, algunsmesesapósoinício dateraplaespecífica paratuberculose. Notarqueas lesóes praticamente desapareceram

Aumento da PIO, e alterações nas estruturas do seguimento anterior não foram vistos em nenhum paciente.

A incidência de lesões de FO compatíveis com tuberculose foi de 5,5\% (tabela 2). Quando considerados somente os pacientes com tuberculose miliar, os co-infectados com HIV e aqueles internados, a incidência foi de $40 \%, 33,5 \%$ e $9 \%$ respectivamente (tabelas 2 e 3 ). Estes achados mostram que existem fatores relacionados à tuberculose que podem facilitar a presença de lesões oculares.
A idade dos pacientes com lesões de FO variou entre 25 e 81 anos, com média de 41,88 anos ( $\mathrm{dp}=16,44)$. Dos 16 casos, 12 (75\%) eram do sexo masculino e $4(25 \%)$, do feminino. A baciloscopia foi positiva em $9(56,3 \%)$ pacientes. Nove $(56,3 \%)$ pacientes residiam no DF, enquanto $7(43,8 \%)$ vieram de outros estados para realizar o tratamento. Treze $(81,3 \%)$ pacientes estavam realizando o tratamento pela primeira vez e $3(18,8 \%)$ eram retratamento. O tempo de sintomas variou entre zero e 48 semanas, com média de 12,86 $(\mathrm{dp}=12,79)$ semanas. Estas variáveis não apresentaram associação estatística com a variável lesão de fundo de olho.

Dos 16 casos, $13(81,2 \%)$ estavam internados e $3(18,8 \%)$ faziam tratamento ambulatorial. Este achado mostra a importância do fator internação e, portanto, gravidade da doença, para o aparecimento das lesões. No estudo de Biswas, Badrinath (1995), a morbidade ocular em pacientes com tuberculose sistêmica foi de 1,39\%; no entanto, não foram avaliados pacientes com hemoptise e dispnéia. A baixa incidência de lesões oculares tuberculosas, observada por estes autores, pode ter ocorrido devido à exclusão destes pacientes ${ }^{(18)}$.

Seis $(38 \%)$ pacientes com lesões de FO estavam co-infectados pelo vírus do HIV e pelo M. tuberculosis. Bouza et al. (1997) encontraram uma incidência de $26,4 \%$ de alterações oculares em pacientes co-infectados com HIV e tuberculose ${ }^{(2)}$. Campinchi-Tardy et al (1994) e outros autores consideram que o achado de nódulos de coróide em pacientes co-infectados pelo M. tuberculosis e pelo HIV, sugere a possibilidade de tuberculose disseminada ${ }^{(7-8,10)}$.

A tuberculose miliar esteve presente em $38 \%$ dos pacientes com lesões de fundo de olho, concordando com os achados de Bouza et al (1997), que identificaram a tuberculose miliar como sendo fator de risco para o envolvimento ocular ${ }^{(2)}$, e com os achados de trabalhos anteriores que já demonstravam a freqüente relação entre esta entidade clínica e lesões de coróide ${ }^{(11-12,17)}$.

A localização pulmonar isolada da tuberculose esteve presente em $9(56 \%)$ casos e $1(6 \%)$ paciente apresentou localização extrapulmonar. No entanto, a análise multivariada não mostrou associação estatística entre estas variáveis e lesão de FO, concordando com Bouza et al. (1997) e com Massaro et al. (1964), que encontraram lesões oculares tuberculosas em pacientes com tuberculose pulmonar e extrapulmonar sem disseminação miliar, em uma proporção de casos não estatisticamente significativa ${ }^{(2,17)}$.

Com relação aos fatores de risco, a presença do HIV $(\mathrm{p}=0,03)$, a localização miliar $(\mathrm{p}=0,09)$ e a internação $(\mathrm{p}=0,09)$ dos pacientes estiveram associadas, e atuaram de maneira independente sobre a presença da lesão ocular. Quando todas

\begin{tabular}{|c|c|c|c|c|}
\hline Tuberculose & Sem lesão FO & Comlesão FO & Total & \% comlesão FO \\
\hline Pulmonar & 228 & 9 & 237 & 3,8 \\
\hline Extrapulmonar & 39 & 1 & 40 & 2,5 \\
\hline Total & 276 & 16 & 292 & 5,5 \\
\hline
\end{tabular}




\begin{tabular}{|lcccc|}
\hline \multicolumn{4}{|c|}{ Tabela 3. Incidência de lesões defundo de olho (FO) portuberculose, quando considerados somente pacientes com HIV ou intemados } \\
& Sem lesão FO & Com lesão FO & Total & \% com lesão FO \\
HIV & 12 & 6 & 18 & 33,5 \\
Intemados & 135 & 13 & 148 & 9,0 \\
\hline
\end{tabular}

essas características estiveram presentes, a probabilidade do paciente apresentar lesão de FO foi de 0,66 (66\%).

Depois de ajustados os efeitos das variáveis restantes, os pacientes tuberculosos portadores de HIV tiveram 5,05 mais chances de desenvolver lesão de fundo de olho que pacientes tuberculosos considerados não portadores de HIV. Pacientes com tuberculose miliar tiveram 8,13 mais chances de contrair a lesão que aqueles com tuberculose extrapulmonar, e os tuberculosos internados, 3,27 mais chances de apresentar essas alterações que os não internados (tabela 4).

Estes achados nos levaram a acreditar que os pacientes internados em enfermarias de tisiologia portadores de formas graves da doença como a tuberculose miliar e/ou co-infectados com HIV, têm maior chance de apresentar lesões oculares por tuberculose do que pacientes em tratamento ambulatorial, sem indicação de internação. Isto deve ocorrer provavelmente devido à disseminação hematogênica do bacilo facilitada pelo estado geral comprometido e queda do sistema imunológico destes pacientes.

Sendo o FO um dos únicos locais do organismo onde o tubérculo pode ser observado em exame clínico, a fundoscopia assume especial importância, principalmente naqueles pacientes com infecção por HIV em estágios avançados, onde o quadro clínico da doença é bastante atípico e a baciloscopia, freqüentemente negativa ${ }^{(6)}$; e nos pacientes com tuberculose miliar, onde as lesões pulmonares são intersticiais, dificultando o isolamento do bacilo ${ }^{(1)}$. Nesses casos, o exame de fundo de olho deve preceder procedimentos diagnósticos invasivos ${ }^{(12,17)}$.

\section{O N C L U S Ã O}

Diante destes resultados, concluímos que na avaliação da incidência de lesões de FO em pacientes com tuberculose, devem ser levadas em consideração certas características individuais, visto que existem fatores de risco para a presença destas lesões.

Sugerimos então, exame oftalmológico em pacientes com tuberculose sistêmica nos seguintes casos: 1) qualquer paciente tuberculoso com queixas de baixa visual, pela possibilidade de

\begin{tabular}{|cc|}
\hline $\begin{array}{c}\text { Tabela 4. Odds ratio com intervalo de confiança (IC) de } 90 \% \\
\text { relativos à presença do HIV, à tuberculose miliare à internação }\end{array}$ \\
Variável & Odds ratio com IC $90 \%$ \\
\hline HN & $5,05(1,48 ; 17,17)$ \\
Tuberculosemiliar & $\mathbf{8 , 1 3}(1,06 ; 62,40)$ \\
Intemação & $3,27(1,02 ; 10,53)$ \\
\hline
\end{tabular}

apresentar lesões destrutivas oculares; 2) pacientes co-infectados pelo M. tuberculosis e pelo HIV, para se avaliar a possibilidade de tuberculose disseminada; 3 ) pacientes com suspeita de tuberculose miliar, como auxílio diagnóstico; 4) pacientes com AIDS e tuberculose miliar, que estejam internados, pela alta probabilidade de apresentar lesões de fundo de olho.

A B S T R A C T

Purpose: To evaluate the incidence of eye posterior lesions in patients with tuberculosis in the Federal District, Brazil, and to identify the risk factors for the appearance of these lesions. Methods: In a prospective study, indirect binocular ophthalmoscopy was performed in 292 patients with systemic tuberculosis. These patients were examined in five different Regional Hospitals of the Federal District, between August $1^{\text {st }}, 1997$ and July $30^{\text {th }}, 1998$. A multiple analysis was applied to identify associations between the variable "eye posterior lesion" and the variables "sex, age, bacilloscopy, HIV, tuberculosis site, patient hospitalization, duration of symptoms, origin of the patient and previous experience with treatment. Results: The results showed a 5,5\% incidence of posterior lesions in patients with tuberculosis. A multiple analysis showed that HIV coinfection, patient hospitalization and miliary tuberculosis were risk factors for the presence of eye posterior lesions. When these characteristics were simultaneously present, the probability of a patient to present these lesions was $66 \%$. Conclusions: In the evaluation of the incidence of eye posterior lesions in patients with tuberculosis, certain individual characteristics should be taken in to account, since risk factors, such as HIV co-infection, miliary tuberculosis, and hospitalization, influenced the presence of these lesions.

Keywords: Tuberculosis/complications; Fundus oculi; Choroid; Ocular tuberculosis; Miliary tuberculosis; Acquired immunodeficiency syndrome

REFERÊNCIAS

1. Brasil. Ministério da Saúde: Campanha Nacional Contra Tuberculose. Controle da tuberculose: uma proposta de integração ensino serviço. Brasília; 1994.

2. Bouza E, Merino P, Munõz P, Sanchez-Carrillo C, Yanes J, Cortés C. Ocular tuberculosis: a prospective study in a general hospital. Medicine 1997;76:53-61.

3. Donahue HC. Ophthalmologic experience in a tuberculosis sanatorium. Am J Ophthalmol 1967;64:742-8.

4. Duke Elder S, Abrams D. Diseases of uveal tract. In: Duke Elder. System of Oftalmology. St. Louis: CV Mosby; 1970. p.246-84.

5. Rosemberg, J, Tarantino AB. Tuberculose. In: Tarantino AB. Doenças pulmonares. Rio de Janeiro: Guanabara Koogan; 1997. p.323-417. 
6. Organização Mundial da Saúde. TB/HIV: Manual clínico para o Brasil. Geneva, OMS; 1998.

7. Blodi BA, Johnson MW, McLeish WM, Gass JD. Presumed choroidal tuberculosis in a human imunodeficiency virus infected host. Am J Ophthalmol 1989;108:605-7.

8. Campinchi-Tardy F, Darwiche A, Bergmann JF, Chedin P, Nemeth J, Campinchi R et al. Bouchut tubercles and AIDS. J Fr Ophtalmol 1994; 17:548-54.

9. Muccioli C, Belfort R. Presumed ocular and central nervous system in a patient with the Acquired Immunodeficiency Syndrome. Am J Ophthalmol 1996;121:217-9.

10. Perez Blazquez E, Montero Rodriguez M, Mendez Ramos MJ. Tuberculous choroiditis and acquired immunodeficiency syndrome. Ann Ophthalmol 1994; 26:50-4.
11. Illingworth RS, Wrigth T. Tubercles of the choroid. Br Med J 1948;2:365-8.

12. Olazabal F. Choroidal tubercles: a neglected sign. JAMA 1967;200:374-7.

13. Tejada P, Mendez M.J, Negreira S. Choroidal tubercles with tuberculous meningitis. Int Opththalmol 1994;18:115-8.

14. Jabbour NM, Farias B, Trempe CL. A case of pulmonary tuberculosis presenting with a choroidal tuberculoma. Ophthalmology 1985;92:834-7.

15. Lyon CE, Grimson BS, Peifer RL, Merritt JC. Clinicopathological correlation of a solitary choroidal tuberculoma. Ophthalmology 1985;92:845-50.

16. Rosen, PH, Spalton DJ, Graham EM. Intraocular tuberculosis. Eye 1990; 4(Pt 3):486-92.

17. Massaro D, Katz S, Sachs M. Choroidal tubercles: a clue to hematogenous tuberculosis. Ann Int Med 1964;60:231-41.

18. Biswas J, Badrinath SS. Ocular morbidity in pacients with active systemic tuberculosis. Int Ophthalmol 1995;19:293-8.

\section{Congresso Maranhense de Oftalmologia}

\section{A 21 DE J UN HO DE 2003 SÃO LUIZ - MA}

\section{PRO MO ÇÃO : \\ Sociedade Maranhense de 0 ftalmologia}

IN FO RMAÇÕ ES: Tel.: (98) 235-5353 com Dr. A fonso Reis Farias

e-mail: afonso@medicina.elo.com.br 